\title{
Fungal glucosylceramides: from structural components to biologically active targets of new antimicrobials
}

\section{Leonardo Nimrichter * and Marcio L. Rodrigues}

Laboratório de Estudos Integrados em Bioquímica Microbiana, Instituto de Microbiologia Professor Paulo de Góes, Universidade Federal do Rio de Janeiro, Rio de Janeiro, Brazil

\section{Edited by:}

Joshua D. Nosanchuk, Albert Einstein College of Medicine, USA

\section{Reviewed by:}

Joshua D. Nosanchuk, Albert Einstein

College of Medicine, USA

Attila Gacser, University of Szeged,

Hungary

\section{${ }^{*}$ Correspondence:}

Leonardo Nimrichter, Instituto de

Microbiologia Professor Paulo de

Góes, Universidade Federal do Rio de

Janeiro, Centro de Ciencias da Saude,

Bloco E, sub-solo. 21941590 Rio de

Janeiro, Brazil.

e-mail: nimrichter@micro.ufr.jbr
The first work reporting synthesis of glucosylceramide (cerebrin, GlcCer) by yeasts was published in 1930. During approximately 70 years members of this class of glycosphingolipids (GSL) were considered merely structural components of plasma membrane in fungi. However, in the last decade GlcCer was reported to be involved with fungal growth, differentiation, virulence, immunogenicity, and lipid raft architecture in at least two human pathogens. Fungal GlcCer are structurally distinct from their mammalian counterparts and enriched at the cell wall, which makes this molecule an effective target for antifungal activity of specific ligands (peptides and antibodies to GlcCer). Therefore, GSL are promising targets for new drugs to combat fungal diseases. This review discusses the most recent information on biosynthesis and role of GlcCer in fungal pathogens.

Keywords: glucosylceramide, fungal pathogens, antifungal targets

\section{INTRODUCTION}

The family of glycosphingolipids (GSL) combines a diversity of molecules consisting of at least one saccharide unit covalently linked to a ceramide (Schnaar et al., 2009). The biosynthesis of GSL is complex and involves a repertoire of enzymes that must be carefully expressed and distributed over endoplasmic reticulum (ER) and Golgi in all eukaryotic cells (Halter et al., 2007; Daniotti and Iglesias-Bartolome, 2011). Due to their structural properties and primary cellular location, GSL were initially described as architectural components of cell membranes, straightly connected with fluidity and stability (Feinstein et al., 1975; Tinker et al., 1976; Aaronson and Martin, 1983; Campanella, 1992). Recent finding, however, clearly demonstrate that their role goes beyond the former concepts, since these molecules are major components of specialized membrane domains called lipid rafts (Bagnat et al., 2000; Hakomori, 2003, 2008; Wachtler and Balasubramanian, 2006). In addition, GSL have been characterized as relevant structures in events of cell-cell interaction, cell signaling and protein sorting (Bagnat et al., 2000; Bagnat and Simons, 2002; Nimrichter et al., 2008; Lopez and Schnaar, 2009; Schnaar et al., 2009; Staubach and Hanisch, 2011).

In fungal organisms two types of GSL have been reported. Inositol phosphorylceramides (IPC) are used to make complex fungal GSL and have been extensively studied in the yeast model Saccharomyces cerevisiae (Sugimoto et al., 2004; Dickson et al., 2006; Dickson, 2008). They consist of a ceramide usually containing phytosphingosine as long chain base, associated with a very long fatty acid $\left(\mathrm{C}_{24: 1}-\mathrm{C}_{26: 1}\right)$. A unit of inositol is covalently linked to the ceramide and the preformed molecule, which is not found in mammalian cells, is the substrate for mannosyltransferases for the synthesis of mannosylinositol phosphorylceramide (MIPC) and mannosyldiinositol phosphorylceramide $\left[\mathrm{M}(\mathrm{IP})_{2} \mathrm{C}\right]$. The second major class of GSL synthesized by fungal cells is the monohexosylceramide (CMH, glycosylceramide, cerebroside, or cerebrin). The sugar moiety covalently linked to a distinct ceramide is usually glucose (GlcCer) or, to a minor extent, galactose (GalCer; Boas et al., 1994; Takahashi et al., 1996; Levery et al., 2000, 2002; Rodrigues et al., 2000; Toledo et al., 2000; Pinto et al., 2002; Barreto-Bergter et al., 2004; da Silva et al., 2004; Nimrichter et al., 2004, 2005a). GlcCer and GalCer are not synthesized by S. cerevisiae but are part of the lipid arsenal of classical fungal pathogens, excepting Candida glabrata (Saito et al., 2006; Tavares et al., 2008).

A very conserved structure was described as the major GlcCer in fungal pathogens, the $N-2^{\prime}$-hydroxy(hexa/octa)de(ca/ce)noyl-1beta-D-glucopyranosyl-9-methyl-4,8-sphingadienine. GlcCer distribution is not limited to fungal membranes, since large amounts of this GSL have been also found at cell wall (Rodrigues et al., 2000; Nimrichter et al., 2005a; Rhome et al., 2011). Although GlcCer have been identified as fungal components decades ago, knowledge about their functions during fungal growth/dimorphism, lipid raft formation, and correlation with virulence has been recently reported. In this review we discuss the most recent findings that characterize the functions of GlcCer and its biosynthetic steps as potential targets for new antifungal drugs. The putative correlation between GlcCer, lipid rafts and secretion and the potential role of this GSL as virulence regulators are also reviewed.

THE UNCOVERED PATHWAY FOR FUNGAL GIcCer SYNTHESIS Sphingoid bases of fungal GlcCer are structurally distinct from mannosylated IPCs and from their counterparts in animal cells. They have a methyl group at C-9 and an extra unsaturation at C-8, forming the typical fungal base mentioned above (for review please see Barreto-Bergter et al., 2004). However, initial steps of ceramide biosynthesis are the same independent of the cell type studied. 
Ceramide synthesis always begins with condensation of palmitoylCoA and serine, followed by sphinganine formation through the activity of a 3-ketosphinganine reductase (Barreto-Bergter et al., 2004; Li et al., 2006; Schnaar et al., 2009; Gault et al., 2010). The product, sphinganine, is then $N$-acylated to generate ceramide. Two distinct pools of ceramide operate as building blocks for major fungal GSL (CMH and IPCs). They differ according to the hydroxylation level of long chain base (dihydroxy or trihydroxy) and the extension of hydroxylated fatty acids $\left(\mathrm{C}_{16: 1}-\mathrm{C}_{18: 1}\right.$ or $\mathrm{C}_{24: 1}-$ $\mathrm{C}_{26: 1}$ )(Barreto-Bergter et al., 2004; Dickson et al., 2006; Takakuwa et al., 2008; Rittenour et al., 2011; Ternes et al., 2011). All enzymes involved in fungal ceramide synthesis operate specifically according to their substrate leading to precise production of GlcCer or MIPCs. So far, the only exceptions appear to be Magnaporthe grisea and Neurospora crassa, discussed later on (Lester et al., 1974; Maciel et al., 2002). It implies that preformed ceramide must be considerably modified before GlcCer or IPC synthesis. This section is focused on fungal GlcCer biosynthesis. Approaches utilized for structural characterization of these molecules are discussed in detail by Barreto-Bergter et al. (this issue).

Synthesis of IPC and GlcCer must include a branching point, since the enzymes required for this process make part of common and specific biosynthetic pathways. A very elegant study published by Ternes et al. (2011) contributed significantly to the understanding of GlcCer biosynthesis. Briefly, these authors demonstrated that in Pichia pastoris, similarly to S. cerevisiae, Lag1 and Lac1 appear to be the genes responsible for coding the enzymes that make trihydroxy ceramides carrying very long chain fatty acids (Ternes et al., 2011). On the other hand, BarA (biocontrol agent resistance) is related to synthesis of dihydroxy ceramides bearing hydroxylated $\mathrm{C}_{16}-\mathrm{C}_{18}$ fatty acids in P. pastoris. BarA was originally identified by a screen assay performed to isolate mutants from $A$. nidulans resistant to the polyketide HSAF, a heat-stable antifungal factor isolated from the biocontrol agent Lysobacter enzymogenes (Zhang and Yuen, 1999). In A. nidulans, exposure to HSAF leads to a rapid loss of the formin SepA at growing hyphal tips and disturbs the polarized growth (Li et al., 2006). Phylogenetic analysis indicated that BarAp belongs to a distinct clade of ceramide synthases when compared to Lag1p and Laclp. Accordingly, $A$. nidulans lacking BarAp do not synthesize GlcCer (Li et al., 2006). In Kluyveromyces lactis, overexpression of Lac 1 increased GlcCer synthesis while gene disruption blocked this pathway, leading to IPC accumulation (Takakuwa et al., 2008). Disruption of Lag 1 appears to be lethal in K. lactis. Orthologous expression of Lac1 from $K$. lactis in $S$. cerevisiae resulted in IPC with $\mathrm{C}_{18: 1}$ fatty acids, which are presumably exclusive to GlcCer. Sequence analysis suggests that Lac1 of $K$. lactis belongs to the same clade as BarA (Rittenour et al., 2011). These data indicated that when BarA is absent in fungi that express GlcCer, $L a c 1$ is the gene involved with GlcCer production.

The biosynthetic steps occurring right after ceramide hydroxylation at C-2 of fatty acid are $\Delta 4$-desaturation, $\Delta 8$-desaturation, C-9-methylation, and then glucose addition by ceramide glucosyl ceramide-synthetase (GCS; Ternes et al., 2002, 2011; Michaelson et al., 2009). Membrane-bound desaturases are part of a superfamily defined by the histidine boxes $\mathrm{HX} 3-4 \mathrm{H}, \mathrm{HX} 2-3 \mathrm{HH}$, and $(\mathrm{H} / \mathrm{Q}) X 2-3 \mathrm{HH}$ that usually require $\mathrm{NAD}(\mathrm{P}) \mathrm{H}$ and $\mathrm{O}_{2}$ as cofactors
(Sperling et al., 1995; Zauner et al., 2008). Searching proteins with histidine box sequence motifs a family of $\Delta 4$-desaturase was discovered. Homo sapiens, Mus musculus, Drosophila melanogaster, and Candida albicans $\Delta 4$-desaturases were expressed in S. cerevisiae and biochemically characterized (Ternes et al., 2002). Sphing-4-enine and 4-hydroxysphinganine were characterized as products in all situations, indicating a bifunctional activity $(\Delta 4$ desaturase and C14-hydroxylase). This enzymatic step appears to be essential, given that $P$. pastoris mutant lacking $\Delta 4$-desaturase is not able to make GlcCer (Ternes et al., 2011). Insertion of a double bond between C-8 and C-9 of long chain base, the step catalyzed by a $\Delta 8$-desaturase, does not appear to be a requirement for GlcCer. $P$. pastoris lacking the enzyme is still able to build regular amounts of GlcCer.

Transference of the C-9-methyl group to the sphingoid base is the last step of ceramide synthesis used to construct fungal GlcCer. The enzyme responsible for this step belongs to the superfamily of $S$-adenosylmethionine-(SAM)-dependent methyltransferases, appears to be membrane-bound and requires a $\Delta 4,8$-desaturated ceramide as substrate (Ternes et al., 2006). As observed for $\Delta 8$ desaturase, absence of C-9 methyltransferase in P. pastoris (coding the enzyme Ppmt1) does not impair GlcCer synthesis, resulting in the exclusive formation of methylated ceramide. In C. albicans, strains lacking GlcCer, or C-9 methyltransferase were also viable, although a deficient hyphal growth was observed when compared to the wild type strain (Oura and Kajiwara, 2010). Two genes encoding putative C-9 methyltransferase were found in Fusarium graminearum, FgMT1, and FgMT2 (Ramamoorthy et al., 2009). Both genes complemented the lack of C-9 methyltransferase activity in P. pastoris mutant Ppmt1. FgMT1 disruption did not change the ceramide methylation and mutants grew as wild type cells. On the other hand, disruption of FgMT2 reduced the enzymatic product in approximately $35 \%$, decreased growth ratio, altered shape, and size of conidia, reduced mycelial formation and virulence to A. thaliana leaves and wheat. Double-knockouts were not viable, suggesting that C-9 methyltransferase is essential to fungal growth in F. graminearum.

Glucose transfer to ceramide occurs at Golgi stacks in animal cells through a CGS (Halter et al., 2007). A fraction of newly synthesized GlcCer returns to ER and is transported to Golgi lumen to synthesize LacCer and other complex GSL. Using a second pathway, intact GlcCer reaches the cell surface through a non-vesicular pathway. In yeast, ceramide is also transported to Golgi through vesicular and non-vesicular routes (Funato and Riezman, 2001) where complex GSL will be synthesized. It is not clear whether $N$-2' -hydroxy(hexa/octa)de(ca/ce)noyl-1-betaD-glucopyranosyl-9-methyl-4,8-sphingadienine follows a similar pathway.

The complexity of fungal GSL biosynthesis is supported by studies on the phytopathogens F. graminearum and M. grisea. F. graminearum is a causative agent of head blight, a plant disease that results in crop loss or grain unsuitability for animal consumption due to mycotoxin production ( $\mathrm{Xu}$ and Nicholson, 2009). As observed for A. nidulans, Barl mutants of F. graminearum do not synthesize GlcCer and are resistant to HSAF (Rittenour et al., 2011). GlcCer was also isolated from lipid extracts of the rice blast agent M. grisea (Maciel et al., 
2002). The typical fungal GlcCer structure was characterized in this model, consisting of $N-2^{\prime}$-hydroxy(octa/hexa)decanoyl-1-O$\beta$-D-glucopyranosyl-9-methyl-4,8-sphingadienine. The $M$. grisea crude extract showed an extra orcinol positive TLC band migrating above GlcCer, indicative of a dihexosylceramide (CDH). The purified molecule was identified as lactosylceramide (LacCer). Unusually, the lipid moiety of LacCer consisted of a phytosphingosine covalently linked to a $\mathrm{C}_{24: 2}$ fatty acid. A tetrahexosylceramide has been also reported in N. crassa (Lester et al., 1974). By using gas and thin layer chromatography the identified components were $\mathrm{Gal}_{3} \mathrm{Glc}$, sphinganine, and a $\mathrm{C}_{24: 1}$ fatty acid.

These results establish that GSL biosynthesis is conserved in fungal cells; however, it cannot be summarized by a simple pathway. It seems important to consider that different species must be investigated individually. Reconstituted strains on GSL pathways also should be carefully investigated, since proper enzyme location usually cannot be assured, which may result in unusual products.

\section{CORRELATION BETWEEN FUNGAL GIcCer, LIPID RAFTS, AND SECRETION MECHANISMS}

To our knowledge, the mechanisms displayed by fungal organisms to traffic GlcCer intracellularly have never been investigated. Fungal GlcCer destiny includes plasma membrane and cell wall (Rodrigues et al., 2000; Nimrichter et al., 2005a), as well as the extracellular space (Rodrigues et al., 2007a, 2008a,b; Nosanchuk et al., 2008).

As mentioned previously, along with sterol and GPI-anchored proteins, GSL are components of lipid domains or lipid rafts (Hakomori, 2003, 2008). These lipid platforms are found in different cell types, including non-pathogenic and pathogenic fungi, where they participate in polarization and compartmentalization steps of the plasma membrane (Bagnat et al., 2000; Bagnat and Simons, 2002; Martin and Konopka, 2004; Siafakas et al., 2006; Wachtler and Balasubramanian, 2006). In the C. albicans model, the ability of fungal cells to switch from yeast to hyphae is considered a crucial step for host cell colonization (Mitchell, 1998). Such morphological changes are dependent on a polarized growth mediated by cytoskeleton (Whiteway and Bachewich, 2007). The first correlation between lipid rafts and polarization in C. albicans was suggested by Martin and Konopka (2004). They observed accumulation of sterols at the growing tip of hyphal cells and at sites of septum formation in mature hyphae. In addition, treatment with myriocin, an inhibitor of serine palmitoyltransferase, decreases filipin staining, and reduces Pmalp recruitment to lipid rafts. Pmalp is a plasma membrane proton pump that is considered a raft marker in C. albicans and S. cerevisiae (Bagnat et al., 2001; Insenser et al., 2006). Along with Pmalp, heat shock proteins (HSP70, HSP90) and molecules required for sterol metabolism, energy production, and polysaccharide synthesis were compartmentalized in lipid domains in C. albicans (Insenser et al., 2006). A connection between polarized growth, lipid rafts, and GlcCer was also observed in F. graminearum and A. nidulans lacking Barlp and GlcCer as well. In F. graminearum, germ tube formation occurs regularly in the absence of Barlp, but hyphal tip organization is considerably altered. Although sterols accumulate in hyphal tips of a wild type strain, $\Delta$ bar 1 mutants lack this distribution. Similarly, no filipin staining was visualized at hyphal tips of A. nidulans lacking Barlp. These mutants do not display regular Spitzenkorper when stained with FM4-64, a fluorescent lipid marker. Polarization defects were confirmed by the altered tropomyosin distribution. Together, these data suggested a requirement of GlcCer and intact lipid rafts for hyphal growth and polarization of fungal cells.

Lipid rafts were also isolated from the opportunistic pathogen C. neoformans. GlcCer, phospholipase B1 (PLB1), and $\mathrm{Cu} / \mathrm{Zn}$ superoxide dismutase (SOD1), molecules involved with fungal virulence, were characterized as raft components in this fungus (Siafakas et al., 2006). It was hypothesized that membranes of $C$. neoformans contain lipid domains that concentrate some virulence factors at fungal surface. Secretion of virulence factors could be associated to their aggregation to lipid rafts.

A few years ago, we discussed aspects of cell wall that, at that point, were considered unusual (Nimrichter et al., 2005b). We then raised the possibility that compact vesicles capable of crossing polar environments, such as the fungal cell wall, could be used by fungal organisms for the extracellular release of macromolecules. Lipid rafts would be likely necessary to increase vesicle stability. In fact, we recently demonstrated that $C$. neoformans produces extracellular vesicles containing GlcCer, sterol, and SOD1 (Rodrigues et al., 2007a, 2008a). Trans-cell wall traffic of GlcCer-containing vesicles could in fact explain the high amounts of GlcCer that are found at the cell wall of fungal pathogens (Rodrigues et al., 2000; Nimrichter et al., 2005a). Further studies revealed that extracellular vesicles are produced by different fungal organisms, possibly representing a conserved mechanism of trans-cell wall transport (Nosanchuk et al., 2008; Oliveira et al., 2010a,b). A number of proteins can reach the extracellular environment through these vesicles, including other lipid raft components, such as Pma1p, HSP70, and HSP90 (Albuquerque et al., 2008; Rodrigues et al., 2008a). The putative correlation between extracellular vesicle formation and fungal lipid rafts is then suggested.

Molecular exportation to the extracellular space through vesicles is not limited to proteins. The polysaccharide glucuronoxylomannan (GXM), a major capsule component of C. neoformans, follows a vesicle association pathway to leave the cells, in a process that apparently requires a close association with GlcCer (Rodrigues et al., 2007a; Oliveira et al., 2009). These data indicate that GlcCer regulates/participates on polysaccharide and protein sorting in fungal cells. Such processes involve compartmentalization and changes in membrane shape. This attribute could be correlated with another property of some complex GSL, the capacity to control membrane curvature and vesicle formation (Sonnino et al., 2007). Recently, a correlation between GlcCer and Golgi morphology was observed in plant cells (Melser et al., 2010). Treatment of A. thaliana with the inhibitor of GlcCer synthesis PDMP disturbed transport of soluble and membrane-associated secretory proteins to plant cell surface. This phenotype was accompanied by remarkable morphological changes and protein retention in the Golgi of treated cells. Reduction on Golgi cisternal thickness and stack width together with an increase in the number of vesicular structures surrounding this organelle was visualized after treatment with drugs that inhibit ceramide glycosylation.

As depicted above, pharmacological drugs that inhibit GSL synthesis are important tools to investigate the role of GSL and lipid 
rafts physiological events in animal cells (Tifft and Proia, 2000). Their activity against fungi is controversial, as discussed below.

Treatment of Aspergillus species with D-threo-1-phenyl2-palmitoyl-3-pyrrolidino propanol (P4) and D-threo-3P,4Pethylenedioxy-P4 (EDO-P4), inhibitors of ceramide glycosylation, was followed by a decrease on GlcCer expression and defects in spore germination, cell cycle, and hyphal growth (Levery et al., 2002). Hillig et al. (2005) extended these findings by evaluating the activity of GSL inhibitors on $P$. pastoris expressing heterologous GCS. The activity of these enzymes was measured in cell-free membrane fractions in the presence of EDO-P4, a GCS inhibitor. Although the human enzyme was strongly inhibited by this drug, it showed no effect over fungal GCS (for instance, C. albicans). These results, however, must be carefully interpreted, given the lack of information in the literature on the properties of GCS activity from other fungal species. However, we could not exclude the possibility that other fungal species are sensitive to derivatives of these inhibitors.

\section{GIcCer AND FUNGAL VIRULENCE IN MURINE MODELS}

As discussed above it is unquestionable that GlcCer is a key molecule during physiological events in fungal organisms. However, mutants lacking GCS are usually viable. These mutant strains have been used to evaluate the role of GlcCer during infection. In this section we will debate the capacity of fungal GlcCer to regulate virulence in animal models of mycoses.

The first report showing that GlcCer could influence the capacity of a fungal organism to cause disease was established by Rittershaus et al. (2006). C. neoformans lacking GlcCer synthesis was generated after GCS disruption and used in murine models of cryptococcosis. Although the capacity to produce key virulence factors of $C$. neoformans was intact in the mutant, this strain was avirulent when inhaled by $\mathrm{CBA} / \mathrm{J}$ mice. Dissemination to the brain was not observed even after 90 days of infection. When injected intravenously using the same mouse background, yeasts of $C$. neoformans reached different tissues, including the brain, with a similar outcome observed for WT strain. Histopathological sections confirmed these data and also showed yeast cells confined to granulomas in the lung of mice after intranasal infection. These granulomas were necessary to control fungal growth since brain dissemination was observed after inhalation of GCS mutants by T- and NK-cell-immunodeficient mice, where granuloma formation is impaired (Kechichian et al., 2007). A refined mechanism of yeast cell cycle transition correlated with $\mathrm{pH}$ and $\mathrm{CO}_{2}$ concentration was suggested. For establishment of cryptococcosis, GlcCer appears to be required for fungal growth in the blood and alveolar spaces of the lung, which, in contrast to the acidic intracellular environment in macrophages, have a neutral $\mathrm{pH}$. Corroborating with these data T- and NK-cell-immunodeficient mice depleted of alveolar macrophages had decreased dissemination to the brain and prolonged survival. The hypothesis raised by Rittershaus et al. (2006) is also in agreement with experiments published by Saito et al. (2006). These authors reported a relationship between GlcCer synthesis and the capacity of fungal cells to grow under neutral or alkaline $\mathrm{pH}$.

Results from a meticulous work developed by Noble et al. (2010) using the model C. albicans reinforce the role of GlcCer as a virulence regulator. These authors disrupted 674 genes in C. albicans using auxotrophic markers with no effect on virulence. Mutants were then screened for three major phenotypes: (i) pathogenicity in mouse model of candidiasis, (ii) morphological switching (yeast to hypha conversion), and (iii) cell proliferation in vitro. The experiments in vivo disclosed 115 mutants with impaired pathogenicity. Among these only 15 mutants displayed impairment to differentiate and grow as hypha. In addition, in the group of 115 mutants with impaired pathogenicity, 89 exhibited growth rates that were close to the WT strain. Therefore, delay in fungal growth did not correlate with capacity to cause disease in mice. The authors then analyzed the mutants with impaired capacity to colonize mice but efficient in proliferating and in promoting morphological changes. Strikingly, four mutants were involved with synthesis of GlcCer.

Previous studies by Oura and Kajiwara (2010) have shown that mutants of C. albicans lacking C-9 methyltransferase, GCS, and $\Delta 8$-saturase have growth rates that are similar to those observed in WT cells. Delayed morphological changes were visualized when these mutants were cultivated in spider medium, but hypha formation was observed in all situations. Contrasting with C. neoformans mutants lacking GlcCer, mutants of $C$. albicans isolated by Noble and colleagues were able to grow under neutral and alkaline $\mathrm{pH}$ similar to WT. Thus, a distinct mechanism of virulence regulation is dependent on GlcCer in C. albicans. The authors identified GlcCer as the first molecule specifically required to govern virulence in C. albicans (Noble et al., 2010).

\section{FUNGAL GIcCer AS TARGETS TO NEW ANTIFUNGAL DRUGS}

Together with other groups we have demonstrated the use of antiGlcCer antibodies to prevent fungal differentiation and growth of fungal pathogens. For instance, these antibodies blocks germ tube formation in C. albicans, Colletotrichum gloeosporioides, and Pseudallescheria boydii (Pinto et al., 2002; da Silva et al., 2004). Furthermore, anti-GlcCer antibodies were able to protect mice in a lethal infection by C. neoformans (Rodrigues et al., 2007b). The mechanism of action for these antibodies is still unknown. The enrichment of GlcCer at budding sites in C. neoformans, suggested that the antibodies could interfere with the cell wall biosynthesis and organization as previously described for the lectin WGA (Ciopraga et al., 1999). Alternatively, these antibodies could associate to GlcCer on lipid rafts, impairing cleavage of GPI-anchored proteins, essential to cell wall assembly. Cross linking of components found on lipid rafts by antibodies or ligands could also initiate a cell signaling response as observed in mammalian cells (Landry and Xavier, 2006; Marta et al., 2008; Bingaman et al., 2010). In this context, the only information available was the prominent cellular damage, with organelle destruction, membrane retraction, and increased vacuolization observed in yeasts from C. neoformans treated with anti-GlcCer (Nimrichter et al., 2005b). Some TEM images suggested an apoptotic mechanism, but no biochemical evidences were characterized.

A set of experiments published by Thevissen et al. (2004) strongly suggested that targeting fungal GlcCer could in fact initiate a cell signaling response in fungal organisms (Thevissen et al., 2004; Aerts et al., 2007). These authors demonstrated that a peptide isolated from radish seeds and named RsAFP2 was able to 
specifically bind to fungal GlcCer (Thevissen et al., 2004). The peptide displayed a potent microbicidal activity in micromolar concentrations against fungal species that express GlcCer. The exact region of GlcCer where the peptide binds is not known, but apparently C-9 methylation at ceramide is not required, since mutants where this enzyme was impaired remained susceptible to the peptide (Ramamoorthy et al., 2009). Binding of peptide was followed by ROS production and fungal death (Aerts et al., 2007). The peptide activity was impaired by anti-oxidative molecules, confirming that fungal death was caused by an oxidative activity. Further studies by our group demonstrated a prophylactic activity for RsAFP2 in a murine model of candidiasis (Tavares et al., 2008). The peptide was biologically active against distinct Candida species and its potency was proportional to the amount of GlcCer expressed by the Candida strain tested.

Although the use of current GCS inhibitors is still questionable, a new alternative to decrease fungal GlcCer content in pathogenic fungi was recently proposed by Rhome et al. (2011): the administration of cerezyme $(\mathrm{Cz})$ to mice lethally infected with C. neoformans. $\mathrm{Cz}$ is a human recombinant enzyme that removes glucose from GlcCer. It has been used as an alternative treatment for patients with Gaucher's, disease where hydrolysis of GlcCer is impaired and the GSL accumulates in lysosomes (Charrow, 2009). This enzyme was proven to hydrolyze fungal $\mathrm{GlcCer}$ and reduce its content in C. neoformans in vitro and in vivo. Cz-induced GlcCer decrease was associated with reduction of fungal membrane stability. In a murine model of cryptococcosis $\mathrm{Cz}$ was also able to increase mice survival (Rhome et al., 2011).

\section{CONCLUSION AND PERSPECTIVES}

For over half a century, GlcCer was classified as a structural membrane component of eukaryotic cells. In the last decade,

\section{REFERENCES}

Aaronson, L. R., and Martin, C. E. (1983). Temperature-induced modifications of glycosphingolipids in plasma membranes of Neurospora crassa. Biochim. Biophys. Acta 735, 252-258.

Aerts, A. M., Francois, I. E., Meert, E. M., Li, Q. T., Cammue, B. P., and Thevissen, K. (2007). The antifungal activity of RsAFP2, a plant defensin from Raphanus sativus, involves the induction of reactive oxygen species in Candida albicans. J. Mol. Microbiol. Biotechnol. 13, 243-247.

Albuquerque, P. C., Nakayasu, E. S., Rodrigues, M. L., Frases, S., Casadevall, A., Zancope-Oliveira, R. M., Almeida, I. C., and Nosanchuk, J. D. (2008). Vesicular transport in Histoplasma capsulatum: an effective mechanism for trans-cell wall transfer of proteins and lipids in ascomycetes. Cell. Microbiol. 10, 1695-1710.

Bagnat, M., Chang, A., and Simons, K. (2001). Plasma membrane proton ATPase Pmalp requires raft

however, a number of studies have uncovered sophisticated functions for GlcCer in eukaryotes. In fungi, it is now clear that knowledge on functions related to virulence, growth, and morphological transitions can be connected to specific structural features and particular biosynthetic steps to validate GlcCer as a potential target to development of new antifungal drugs. A major challenge, however, is to identify these drugs. The literature suggests several inhibitors of fungal GlcCer synthesis as candidates. So far, none of them have appeared to be sufficiently specific to allow use in human patients. In parallel, GlcCer ligands are apparently efficient and selective antifungal agents where in fact, peptides and monoclonal antibodies were already administered in murine models with encouraging results. Other candidates, including the antifungal factor HSAF and the enzyme $\mathrm{Cz}$ (which is already approved for use in humans), are also promising GlcCer-binding candidates. We need now more details on how antibodies, peptides, and enzymes interfering with the structure of GlcCer alter the biology of fungal cells, as well as how they impact human/animal physiology. Considering the critical side effects usually observed during antifungal therapy, the low efficacy of currently approved antifungal drugs and the emerging resistance of fungal cells against antifungals, we believe GlcCer is a very promising target for the activity of new compounds with potential to be used in human and animal individuals with severe mycoses.

\section{ACKNOWLEDGMENTS}

Leonardo Nimrichter and Marcio L. Rodrigues were supported by grants from Conselho Nacional de Desenvolvimento Tecnológico (CNPq, Brazil), CAPES, and Fundação Carlos Chagas Filho de Amparo à Pesquisa do Estado do Rio de Janeiro (FAPERJ, Brazil).

of N-2'-hydroxyoctadecanoyl-1-Obeta-D-glucopyranosyl-9-methyl-4, 8 -sphingadienine from species of Aspergillus. Chem. Phys. Lipids 70 11-19.

Campanella, R. (1992). Membrane lipids modifications in human gliomas of different degree of malignancy. J. Neurosurg. Sci. 36, 11-25.

Charrow, J. (2009). Enzyme replacement therapy for Gaucher disease. Expert Opin. Biol. Ther. 9, 121-131.

Ciopraga, J., Gozia, O., Tudor, R., Brezuica, L., and Doyle, R. J. (1999). Fusarium sp. growth inhibition by wheat germ agglutinin. Biochim. Biophys. Acta 1428, 424-432.

da Silva, A. F., Rodrigues, M. L., Farias, S. E., Almeida, I. C., Pinto, M. R., and Barreto-Bergter, E. (2004). Glucosylceramides in Colletotrichum gloeosporioides are involved in the differentiation of conidia into mycelial cells. FEBS Lett. 561, 137-143.

Daniotti, J. L., and Iglesias-Bartolome, R. (2011). Metabolic pathways and intracellular trafficking of gangliosides. IUBMB Life 63, 513-520.

Dickson, R. C. (2008). Thematic review series: sphingolipids. New insights into sphingolipid metabolism and function in budding yeast. J. Lipid Res. 49, 909-921.

Dickson, R. C., Sumanasekera, C., and Lester, R. L. (2006). Functions and metabolism of sphingolipids in Saccharomyces cerevisiae. Prog. Lipid Res. 45, 447-465.

Feinstein, M. B., Fernandez, S. M., and Sha'afi, R. I. (1975). Fluidity of natural membranes and phosphatidylserine and ganglioside dispersions. Effect of local anesthetics, cholesterol and protein. Biochim. Biophys. Acta 413, 354-370.

Funato, K., and Riezman, H. (2001). Vesicular and nonvesicular transport of ceramide from ER to the Golgi apparatus in yeast. J. Cell Biol. 155, 949-959.

Gault, C. R., Obeid, L. M., and Hannun, Y. A. (2010). An overview of sphingolipid metabolism: from synthesis to breakdown. Adv. Exp. Med. Biol. $688,1-23$. 
Hakomori, S. (2003). Structure, organization, and function of glycosphingolipids in membrane. Curr. Opin. Hematol. 10, 16-24.

Hakomori, S. I. (2008). Structure and function of glycosphingolipids and sphingolipids: recollections and future trends. Biochim. Biophys. Acta 1780, 325-346.

Halter, D., Neumann, S., Van Dijk, S. M., Wolthoorn, J., De Maziere, A. M., Vieira, O. V., Mattjus, P., Klumperman, J., Van Meer, G., and Sprong, H. (2007). Pre- and post-Golgi translocation of glucosylceramide in glycosphingolipid synthesis. J. Cell Biol. 179, 101-115.

Hillig, I., Warnecke, D., and Heinz, E. (2005). An inhibitor of glucosylceramide synthase inhibits the human enzyme, but not enzymes from other organisms. Biosci. Biotechnol. Biochem. 69, 1782-1785.

Insenser, M., Nombela, C., Molero, G., and Gil, C. (2006). Proteomic analysis of detergent-resistant membranes from Candida albicans. Proteomics 6(Suppl. 1), S74-S81.

Kechichian, T. B., Shea, J., and Del Poeta, M. (2007). Depletion of alveolar macrophages decreases the dissemination of a glucosylceramide-deficient mutant of Cryptococcus neoformans in immunodeficient mice. Infect. Immun. 75, 4792-4798.

Landry, A., and Xavier, R. (2006). Isolation and analysis of lipid rafts in cell-cell interactions. Methods Mol. Biol. 341, 251-282.

Lester, R. L., Smith, S. W., Wells, G. B., Rees, D. C., and Angus, W. W. (1974). The isolation and partial characterization of two novel sphingolipids from Neurospora crassa: di(inositolphosphoryl)ceramide and ((gal)3glu)ceramide. J. Biol. Chem. 249, 3388-3394.

Levery, S. B., Momany, M., Lindsey, R., Toledo, M. S., Shayman, J. A., Fuller, M., Brooks, K., Doong, R. L., Straus, A. H., and Takahashi, H. K. (2002). Disruption of the glucosylceramide biosynthetic pathway in Aspergillus nidulans and Aspergillus fumigatus by inhibitors of UDP-Glc:ceramide glucosyltransferase strongly affects spore germination, cell cycle, and hyphal growth. FEBS Lett. 525, 59-64.

Levery, S. B., Toledo, M. S., Doong, R. L., Straus, A. H., and Takahashi, H. K. (2000). Comparative analysis of ceramide structural modification found in fungal cerebrosides by electrospray tandem mass spectrometry with low energy collision-induced dissociation of $\mathrm{Li}+$ adduct ions. Rapid Commun. Mass Spectrom. 14, 551-563.
Li, S., Du, L., Yuen, G., and Harris, S. D. (2006). Distinct ceramide synthases regulate polarized growth in the filamentous fungus Aspergillus nidulans. Mol. Biol. Cell 17, 1218-1227.

Lopez, P. H., and Schnaar, R. L. (2009). Gangliosides in cell recognition and membrane protein regulation. Curr. Opin. Struct. Biol. 19, 549-557.

Maciel, D. M., Rodrigues, M. L., Wait, R., Villas Boas, M. H., Tischer, C. A., and Barreto-Bergter, E. (2002). Glycosphingolipids from Magnaporthe grisea cells: expression of a ceramide dihexoside presenting phytosphingosine as the long-chain base. Arch. Biochem. Biophys. 405, 205-213.

Marta, C. B., Bansal, R., and Pfeiffer, S. E. (2008). Microglial Fc receptors mediate physiological changes resulting from antibody crosslinking of myelin oligodendrocyte glycoprotein. J. Neuroimmunol. 196, 35-40.

Martin, S. W., and Konopka, J. B. (2004). Lipid raft polarization contributes to hyphal growth in Candida albicans. Eukaryot. Cell 3, 675-684.

Melser, S., Batailler, B., Peypelut, M., Poujol, C., Bellec, Y., Wattelet-Boyer, V., Maneta-Peyret, L., Faure, J. D., and Moreau, P. (2010). Glucosylceramide biosynthesis is involved in Golgi morphology and protein secretion in plant cells. Traffic 11, 479-490.

Michaelson, L. V., Zauner, S., Markham, J. E., Haslam, R. P., Desikan, R., Mugford, S., Albrecht, S., Warnecke, D., Sperling, P., Heinz, E., and Napier, J. A. (2009). Functional characterization of a higher plant sphingolipid Delta4-desaturase: defining the role of sphingosine and sphingosine1-phosphate in Arabidopsis. Plant Physiol. 149, 487-498.

Mitchell, A. P. (1998). Dimorphism and virulence in Candida albicans. Curr. Opin. Microbiol. 1, 687-692.

Nimrichter, L., Barreto-Bergter, E., Mendonca-Filho, R. R., Kneipp, L. F., Mazzi, M. T., Salve, P., Farias, S. E., Wait, R., Alviano, C. S., and Rodrigues, M. L. (2004). A monoclonal antibody to glucosylceramide inhibits the growth of Fonsecaea pedrosoi and enhances the antifungal action of mouse macrophages. Microbes Infect. 6, 657-665.

Nimrichter, L., Burdick, M. M., Aoki, K., Laroy, W., Fierro, M. A., Hudson, S. A., Von Seggern, C. E., Cotter, R. J., Bochner, B. S., Tiemeyer, M., Konstantopoulos, K., and Schnaar, R. L. (2008). E-selectin receptors on human leukocytes. Blood 112, 3744-3752.
Nimrichter, L., Cerqueira, M. D., Leitao, E. A., Miranda, K., Nakayasu, E. S., Almeida, S. R., Almeida, I. C., Alviano, C. S., Barreto-Bergter, E., and Rodrigues, M. L. (2005a). Structure, cellular distribution, antigenicity, and biological functions of Fonsecaea pedrosoi ceramide monohexosides. Infect. Immun. 73, 7860-7868.

Nimrichter, L., Rodrigues, M. L. Rodrigues, E. G., and Travassos, L. R. (2005b). The multitude of targets for the immune system and drug therapy in the fungal cell wall. Microbes Infect. 7, 789-798.

Noble, S. M., French, S., Kohn, L. A., Chen, V., and Johnson, A. D. (2010). Systematic screens of a Candida albicans homozygous deletion library decouple morphogenetic switching and pathogenicity. Nat. Genet. 42 , 590-598.

Nosanchuk, J. D., Nimrichter, L. Casadevall, A., and Rodrigues, M. L. (2008). A role for vesicular transport of macromolecules across cell walls in fungal pathogenesis. Commun. Integr. Biol. 1, 37-39.

Oliveira, D. L., Nakayasu, E. S., Joffe, L. S., Guimaraes, A. J., Sobreira, T. J., Nosanchuk, J. D., Cordero, R. J., Frases, S., Casadevall, A., Almeida, I. C., Nimrichter, L., and Rodrigues, $M$ L. (2010a). Biogenesis of extracellular vesicles in yeast: many questions with few answers. Commun. Integr. Biol. 3, 533-535.

Oliveira, D. L., Nakayasu, E. S., Joffe, L. S., Guimaraes, A. J., Sobreira, T. J., Nosanchuk, J. D., Cordero, R. J., Frases, S., Casadevall, A., Almeida, I. C., Nimrichter, L., and Rodrigues, M. L. (2010b). Characterization of yeast extracellular vesicles: evidence for the participation of different pathways of cellular traffic in vesicle biogenesis. PLOS ONE 5, e11113. doi:10.1371/journal.pone. 0011113

Oliveira, D. L., Nimrichter, L., Miranda, K., Frases, S., Faull, K. F., Casadevall, A., and Rodrigues, M. L. (2009). Cryptococcus neoformans cryoultramicrotomy and vesicle fractionation reveals an intimate association between membrane lipids and glucuronoxylomannan. Fungal Genet. Biol. 46, 956-963.

Oura, T., and Kajiwara, S. (2010). Candida albicans sphingolipid C9-methyltransferase is involved in hyphal elongation. Microbiology 156, 1234-1243.

Pinto, M. R., Rodrigues, M. L., Travassos, L. R., Haido, R. M., Wait, R., and Barreto-Bergter, E. (2002). Characterization of glucosylceramides in Pseudallescheria boydii and their involvement in fungal differentiation. Glycobiology 12 , 251-260.

Ramamoorthy, V., Cahoon, E. B., Thokala, M., Kaur, J., Li, J., and Shah, D. M. (2009). Sphingolipid C-9 methyltransferases are important for growth and virulence but not for sensitivity to antifungal plant defensins in Fusarium graminearum. Eukaryot. Cell 8, 217-229.

Rhome, R., Singh, A., Kechichian, T., Drago, M., Morace, G., Luberto, C., and Del Poeta, M. (2011). Surface localization of glucosylceramide during Cryptococcus neoformans infection allows targeting as a potential antifungal. PLoS ONE 6, e15572. doi:10.1371/journal.pone.0015572

Rittenour, W. R., Chen, M., Cahoon, E. B., and Harris, S. D. (2011) Control of glucosylceramide production and morphogenesis by the Barl ceramide synthase in Fusarium graminearum. PLoS One 6, e19385. doi:10.1371/journal.pone. 0019385

Rittershaus, P. C., Kechichian, T. B., Allegood, J. C., Merrill, A. H. Jr., Hennig, M., Luberto, C., and Del Poeta, M. (2006). Glucosylceramide synthase is an essential regulator of pathogenicity of Cryptococcus neoformans. J. Clin. Invest. 116, 1651-1659.

Rodrigues, M. L., Nakayasu, E. S., Oliveira, D. L., Nimrichter, L., Nosanchuk, J. D., Almeida, I. C., and Casadevall, A. (2008a). Extracellular vesicles produced by Cryptococcus neoformans contain protein components associated with virulence. Eukaryot. Cell 7, 58-67.

Rodrigues, M. L., Nimrichter, L., Oliveira, D. L., Nosanchuk, J. D. and Casadevall, A. (2008b). Vesicular trans-cell wall transport in fungi: a mechanism for the delivery of virulence-associated macromolecules? Lipid Insights 2, 27-40.

Rodrigues, M. L., Nimrichter, L., Oliveira, D. L., Frases, S., Miranda, K., Zaragoza, O., Alvarez, M., Nakouzi, A., Feldmesser, M., and Casadevall, A. (2007a). Vesicular polysaccharide export in Cryptococcus neoformans is a eukaryotic solution to the problem of fungal transcell wall transport. Eukaryot. Cell 6, 48-59.

Rodrigues, M. L., Shi, L., BarretoBergter, E., Nimrichter, L., Farias, S. E., Rodrigues, E. G., Travassos, L. R., and Nosanchuk, J. D. (2007b). Monoclonal antibody to fungal glucosylceramide protects mice against lethal Cryptococcus neoformans infection. Clin. Vaccine Immunol. 14, 1372-1376. 
Rodrigues, M. L., Travassos, L. R., Miranda, K. R., Franzen, A. J., Rozental, S., De Souza, W., Alviano, C. S., and Barreto-Bergter, E. (2000). Human antibodies against a purified glucosylceramide from Cryptococcus neoformans inhibit cell budding and fungal growth. Infect. Immun. 68, 7049-7060.

Saito, K., Takakuwa, N., Ohnishi, M., and Oda, Y. (2006). Presence of glucosylceramide in yeast and its relation to alkali tolerance of yeast. Appl. Microbiol. Biotechnol. 71, 515-521.

Schnaar, R. L., Suzuki, A., and Stanley, P. (2009). "Glycosphingolipids," in Essentials of Glycobiology, Chap. 10, 2nd Edn. Eds A.Varki, R. D.Cummings, J. D. Esko, H. H. Freeze, P. Stanley, C. R. Bertozzi, G.W. Hart, and M. E. Etzler (Cold Spring Harbor, NY: Cold Spring Harbor Laboratory Press).

Siafakas, A. R., Wright, L. C., Sorrell, T. C., and Djordjevic, J. T. (2006). Lipid rafts in Cryptococcus neoformans concentrate the virulence determinants phospholipase $\mathrm{B} 1$ and $\mathrm{Cu} / \mathrm{Zn}$ superoxide dismutase. Eukaryot. Cell 5, 488-498.

Sonnino, S., Mauri, L., Chigorno, V., and Prinetti, A. (2007). Gangliosides as components of lipid membrane domains. Glycobiology 17, 1R-13R.

Sperling, P., Schmidt, H., and Heinz, E. (1995). A cytochrome-b5containing fusion protein similar to plant acyl lipid desaturases. Eur. J. Biochem. 232, 798-805.

Staubach, S., and Hanisch, F. G. (2011). Lipid rafts signaling and sorting platforms of cells and their roles in cancer. Expert Rev. Proteomics 8, 263-277.

Sugimoto, Y., Sakoh, H., and Yamada, K. (2004). IPC synthase as a useful target for antifungal drugs. Curr. Drug Targets Infect. Disord. 4, 311-322.

Takahashi, H. K., Levery, S. B., Toledo, M. S., Suzuki, E., Salyan, M. E., Hakomori, S., and Straus, A. H. (1996). Isolation and possible composition of glucosylceramides from Paracoccidioides brasiliensis. Braz. J. Med. Biol. Res. 29, 1441-1444.

Takakuwa, N., Ohnishi, M., and Oda, Y. (2008). Significance of the KILAC1 gene in glucosylceramide production by Kluyveromyces lactis. FEMS Yeast Res. 8, 839-845.

Tavares, P. M., Thevissen, K., Cammue, B. P., Francois, I. E., BarretoBergter, E., Taborda, C. P., Marques, A. F., Rodrigues, M. L., and Nimrichter, L. (2008). In vitro activity of the antifungal plant defensin RsAFP2 against Candida isolates and its in vivo efficacy in prophylactic murine models of candidiasis. Antimicrob. Agents Chemother. 52, 4522-4525.

Ternes, P., Franke, S., Zahringer, U., Sperling, P., and Heinz, E. (2002). Identification and characterization of a sphingolipid delta 4desaturase family. J. Biol. Chem. 277, 25512-25518.

Ternes, P., Sperling, P., Albrecht, S., Franke, S., Cregg, J. M., Warnecke, D., and Heinz, E. (2006). Identification of fungal sphingolipid C9-methyltransferases by phylogenetic profiling. J. Biol. Chem. 281, 5582-5592.
Ternes, P., Wobbe, T., Schwarz, M., Albrecht, S., Feussner, K., Riezman, I., Cregg, J. M., Heinz, E., Riezman, H., Feussner, I., and Warnecke, D. (2011). Two pathways of sphingolipid biosynthesis are separated in the yeast Pichia pastoris. J. Biol. Chem. 286, 11401-11414.

Thevissen, K., Warnecke, D. C., Francois, I. E., Leipelt, M., Heinz, E., Ott, C., Zahringer, U., Thomma, B. P., Ferket, K. K., and Cammue, B. P. (2004). Defensins from insects and plants interact with fungal glucosylceramides. J. Biol. Chem. 279, 3900-3905.

Tifft, C. J., and Proia, R. L. (2000). Stemming the tide: glycosphingolipid synthesis inhibitors as therapy for storage diseases. Glycobiology 10 1249-1258.

Tinker, D. O., Pinteric, L., Hsia, J. C., and Rand, R. P. (1976). Perturbation of lecithin bilayer structure by globoside. Can. J. Biochem. 54, 209-218.

Toledo, M. S., Levery, S. B., Straus, A. H., and Takahashi, H. K. (2000). Dimorphic expression of cerebrosides in the mycopathogen Sporothrix schenckii. J. Lipid Res. 41 797-806.

Wachtler, V., and Balasubramanian, M. K. (2006). Yeast lipid rafts? - an emerging view. Trends Cell Biol. 16, $1-4$.

Whiteway, M., and Bachewich, C. (2007). Morphogenesis in Candida albicans. Annu. Rev. Microbiol. 61, 529-553.

Xu, X., and Nicholson, P. (2009). Community ecology of fungal pathogens causing wheat head blight. Annu. Rev. Phytopathol. 47, 83-103.
Zauner, S., Zahringer, U., Lindner, B., Warnecke, D., and Sperling, P. (2008). Identification and functional characterization of the 2-hydroxy fatty $\mathrm{N}$-acyl-Delta3(E)-desaturase from Fusarium graminearum. J. Biol. Chem. 283, 36734-36742.

Zhang, Z., and Yuen, G. Y. (1999). Biological control of Bipolaris sorokiniana on tall fescue by Stenotrophomonas maltophilia strain C3. Phytopathology 89, 817-822.

Conflict of Interest Statement: The authors declare that the research was conducted in the absence of any commercial or financial relationships that could be construed as a potential conflict of interest.

Received: 28 September 2011; accepted: 30 September 2011; published online: 20 October 2011.

Citation: Nimrichter $L$ and Rodrigues $M L$ (2011) Fungal glucosylceramides: from structural components to biologically active targets of new antimicrobials. Front. Microbio. 2:212. doi: 10.3389/fmicb.2011.00212

This article was submitted to Frontiers in Fungi and Their Interactions, a specialty of Frontiers in Microbiology.

Copyright (C) 2011 Nimrichter and Rodrigues. This is an open-access article subject to a non-exclusive license between the authors and Frontiers Media $S A$, which permits use, distribution and reproduction in other forums, provided the original authors and source are credited and other Frontiers conditions are complied with. 